\title{
TRATAMENTO COLONOSCÓPICO DE FÍSTULA COLO-CUTÂNEA COM PLUGUE DE COLÁGENO
}

\author{
Colonoscopic treatment of colocutaneous fistula with collagen plug
}

Júlio Cezar Uili COELHO, Guilherme Francisco GOMES, Júlio Japiassu Marinho de MACEDO

Trabalho realizado no Hospital Nossa Senhora das Graças, Curitiba, PR, Brasil
RESUMO - Introdução - Fístulas digestivas permanecem como complicações mais frequentes após operações abdominais. Quando ocorrem, o tempo de internação, custo e a morbimortalidade pós-operatória aumentam. Apesar das várias opções de tratamento, um número significativo de pacientes requerem procedimentos cirúrgicos complexos para fechá-las. O desenvolvimento da endoscopia intervencionista proporcionou nova opção de tratamento para fístulas gastro-intestinais. Relato do Caso - Paciente com fístula colocutânea foi tratada com sucesso com um plugue de colágeno. A injeção percutânea de azul de metileno identificou o orifício interno da fístula no cólon transverso. Aplicação de clips anteriormente realizada não foi bem sucedida. A fístula fechou em poucos dias após a introdução de plugue de colágeno através de um procedimento combinado percutâneo-colonoscopico. Conclusão - O uso de tampões de colágeno por procedimento percutâneo e endoscópico combinado tem a vantagem de permitir a redução do tempo de tratamento da fístula intestinal e pode evitar intervenções cirúrgicas mais complexas.

\section{Correspondência:}

Júlio Coelho

coelhojcu@yahoo.com.br

Fonte de financiamento: não há

Conflito de interesses: não há

Recebido para publicação: 04/08/2009

Aceito para publicação: 24/11/2009

HEADINGS - Collagen. Fistula. Colonoscopy.
ABSTRACT - Background - Digestive fistulas remain as frequent complications after abdominal operations. When they occur, hospitalization time, cost, morbidity and post-operative mortality increase significantly. Despite various treatment options, a significant number of patients require complex surgical procedures to close them. The development of interventional endoscopy has provided new treatment option for gastro-intestinal fistulas. Case report - Patient with colocutaneous fistula was successfully treated with a collagen plug. Percutaneous injection of methylene-blue identified the internal orifice of the fistula in the transverse colon. Previous clips application was unsuccessful. The fistula closed in few days after collagen plug introduction through a combined percutaneous-colonoscopic procedure. Conclusion - The use of plugs of collagen by combined percutaneous and endoscopic procedure has the advantage of allowing reduced time for treatment of intestinal fistulae and may avoid surgical operations.

\section{INTRODUÇÃO}

s fístulas digestivas permanecem como complicações frequentes
após operações abdominais 1,6 . Quando ocorrem, o tempo de
internação, os custos e a morbidade e mortalidade pós-operatória aumentam significativamente ${ }^{5,6}$. Apesar das várias opções de tratamento, um número significativo de pacientes necessita de procedimentos cirúrgicos complexos para o fechamento da fístula. O desenvolvimento da endoscopia intervencionista tem fornecido nova opção de tratamento para as fístulas gastro-intestinais ${ }^{4,5}$. As crescentes inovações técnicas, seja na aparelhagem ou nos materiais envolvidos, ampliaram os limites da abordagem endoscópica. O objetivo deste trabalho é descrever o caso de uma paciente que foi tratada com sucesso através da inserção colonoscópica de um plugue de colágeno no orifício interno de uma fístula crônica de cólon transverso.

RELATO DO CASO

Mulher de 49 anos foi submetida à enucleação de um tumor endócrino de cabeça do pâncreas. Evoluiu com pancreatite aguda e fístula pancreática de alto 
débito no pós-operatório, sendo manejada inicialmente com octreotide e nutrição parenteral total. No $25^{\circ}$ dia do pós-operatório, a drenagem era de $100 \mathrm{~mL}$ ao dia. Foi realizado papilotomia endoscópica e passagem de prótese no ducto pancreático principal por via transpapilar, com o objetivo de ocluir o orifício interno da fístula.

A paciente desenvolveu coleção intra-abdominal e sepse no $28^{\circ}$ dia de pós-operatório, quando foi submetida à drenagem percutânea de coleções epigástricas. A paciente permaneceu internada por dois meses devido à complicações decorrentes da sepse, recebendo alta com dieta oral livre e drenagem diária de cerca de $50 \mathrm{~mL}$ de secreção sero-purulenta pelo orifício da fístula na pele.

Devido à persistência de drenagem de secreção sero-purulenta em torno de $10 \mathrm{~mL}$ ao dia, foi realizada fistulografia, a qual evidenciou trajeto até o cólon transverso (Figura 1).

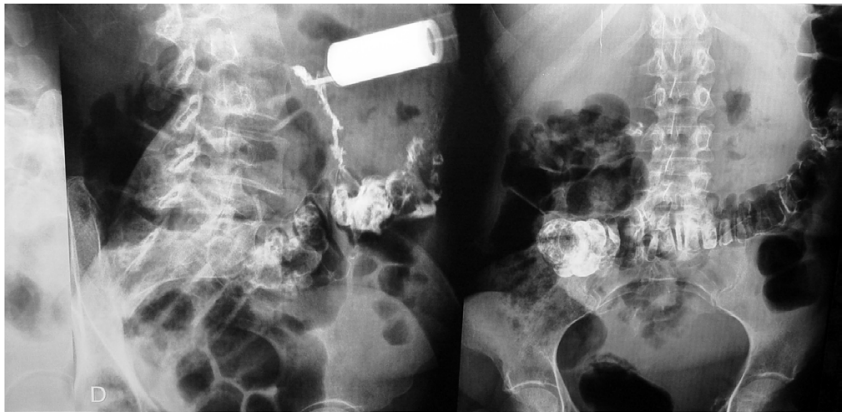

FIGURA 1 - Fistulografia evidenciando uma fístula colocutânea

A injeção de azul de metileno através de um cateter inserido percutaneamente no trajeto fistuloso durante colonoscopia permitiu a identificação do orifício interno no colon transverso. Foram colocados clipes endoscópicos no orifício interno da fístula, sem sucesso pela pouca adaptação deles à mucosa colônica. A presença de tecido granulomatoso endurecido no tecido peri-orificial impediu a fixação dos clipes. Optou-se, então, pela inserção de um segmento de plugue de colágeno $\left(\right.$ Surgisis ${ }^{\circledR}$ ), de dimensões similares as do trajeto fistuloso, pelo orifício interno por via colonoscópica. Para tanto, foi introduzido um cateter de fistulografia (sonda nasogástrica número 8) ao longo de todo o trajeto fistuloso até o seu aparecimento na luz colônica. Posteriormente, uma alça de polipectomia, introduzida dentro da sonda naso-gástrica, auxiliou na tração do plugue para dentro do orifício fistuloso, de modo a obstruí-lo. A drenagem de secreção reduziu rapidamente e em poucos dias o trajeto fistuloso fechou completamente.

\section{DISCUSSÃO}

O manejo das fístulas gastro-intestinais continua a ser um desafio. A maioria fecha com o tratamento

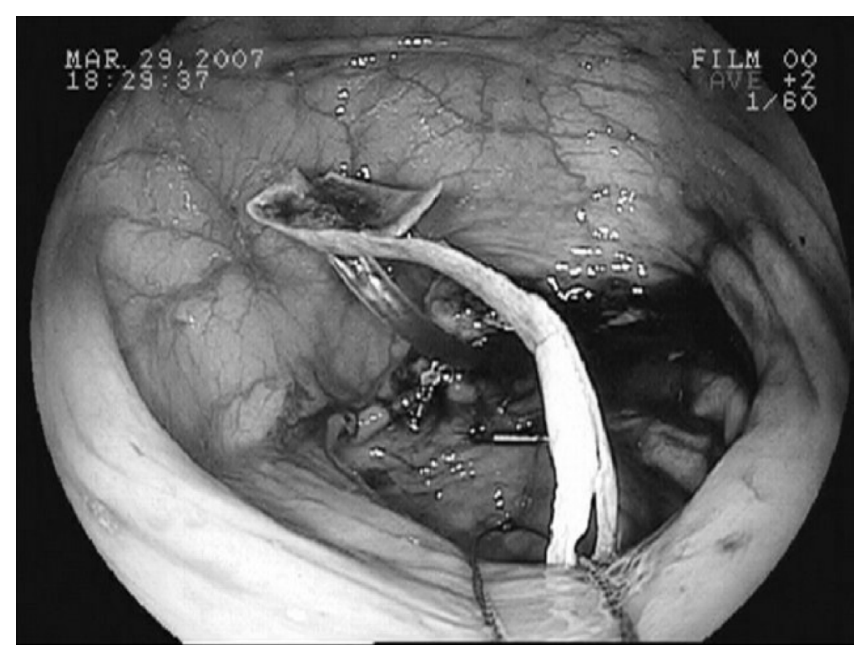

FIGURA 2 - Colonoscopia mostrando o plugue de colágeno sendo tracionado para o interior do orifício interno da fístula colo-cutânea

conservador, constituído basicamente de drenagem completa das secreções, suporte nutricional, inibidores da secreção digestiva e, se necessário, controle do processo infeccioso. Entretanto, este tratamento é prolongado e associado à morbidade e mortalidade potencialmente elevadas. Além do mais, muitos pacientes necessitam tratamento cirúrgico para resolução da fístula.

Os relatos na literatura são crescentes quanto ao uso do tratamento endoscópico para as fístulas gastrointestinais, sejam esofagogástricas ou colo-cutâneas. As matrizes acelulares e os clipes endoscópicos são os materiais mais utilizados para o tratamento endoscópico das fístulas ${ }^{2,3,4}$. Não encontrou-se relato na literatura de tratamento combinado endoscópico e percutâneo.

O material do plugue utilizado é um complexo de colágeno obtido de esqueleto de porco4. Como ele é muito similar ao do homem, uma vez implantado o colágeno se incorpora ao tecido do corpo humano4,5. Após a sua colocação, os tecidos adjacentes produzem vasos sanguíneos e células que rapidamente penetram no plugue e gradualmente o substituem.

\section{CONCLUSÃO}

O uso de plugues de colágeno por via combinada percutânea e endoscópica tem a vantagem de permitir a redução do tempo de tratamento de fístulas digestivas e de evitar procedimentos cirúrgicos em muitos pacientes.

\section{REFERÊNCIAS}

1. Baba RS, Silveira RC, Souza ACP, Paim S, Teixeira MG, Gama AH, Kiss $D$, Cecconello I. Resultado do tratamento das fístulas enterovesicais para doença de Crohn. Rev Bras Colo-Proctol 2008;28:156-9. 
2. Hoyos A, Villegas O, Sanchez JM. Endoloops as a therapeutic option in colocutaneous fistula closure. Endoscopy 2005;37:1258-60,

3. Kim HS, Lee DK, Baik SK. Endoscopic management of colocutaneous fistula after percutaneous endoscopic gastrostomy. Endoscopy 2002; 34:430-3.

4. Maluf-Filho F, Moura E, Sakai P. Endoscopic treatment of esophagogastric fistulae with an acellular matrix. Gastr Endosc 2004; 59:151-3.
5. Maluf-Filho F, Lima MS, Hondo F, Giordano-Nappi JH, Garrido T, Sakai $P$. Experiência inicial no tratamento endoscópico de fístulas gastrocutâneas pós-gastroplastia vertical redutora através de aplicação de matriz acelular fibrogência. Arq Gastroenterol 2008;45:208-11.

6. Von Bahten LC, Nicoluzzi JEL, Silveira FN, Guilherme M, Kumagai LY, Lima VZ. Morbimortalidade da reconstrução de transito intestinal colônica em hospital universitário: análise de 42 casos. Rev Bras Colo-Proctol 2006:26:123-7. 